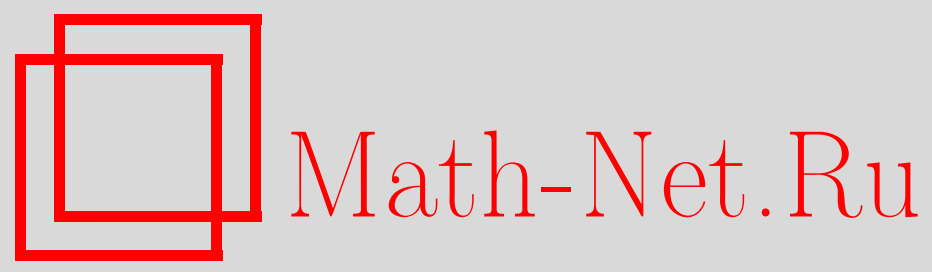

Д. Б. Зотьев, О симплектической геометрии многообразий с почти всюду невырожденной замкнутой 2-формой, $M a$ тем. заметки, 2004, том 76, выпуск 1, 66-77

DOI: https://doi.org/10.4213/mzm95

Использование Общероссийского математического портала Math-Net.Ru подразумевает, что вы прочитали и согласны с пользовательским соглашением http://www . mathnet.ru/rus/agreement

Параметры загрузки:

IP: 44.207 .124 .84

26 апреля 2023 г., 09:09:39 


\title{
О СИМПЛЕКТИЧЕСКОЙ ГЕОМЕТРИИ МНОГООБРАЗИЙ С ПОЧТИ ВСЮДУ НЕВЫРОЖДЕННОЙ ЗАМКНУТОЙ 2-ФОРМОЙ
}

\author{
Д. Б. Зотьев
}

\begin{abstract}
Изучены локальные геометрические свойства многообразий с замкнутой 2-формой, невырожденной в точках всюду плотного собственного подмножества. Введено естественное понятие правильной особой точки, в которой вырождение матрицы формы происходит некоторьм регулярньм образом. В правильных особых точках найдено условие гладкого продолжения гамильтоновой динамической системы, обобщена теорема Дарбу о локальном приведении матрицы формы к каноническому виду, исследовано сингулярное поведение косых градиентов.
\end{abstract}

Библиография: 5 названий.

Рассмотрим $(2 n+2 m)$-мерное симплектическое многообразие $(N, \omega)$ и в нем подмногообразие $M=f^{-1}(b)$, отвечающее некоторому регулярному значению $b \in \mathbb{R}^{2 m}$ гладкого отображения $f: N \rightarrow \mathbb{R}^{2 m}$. Если матрища попарных скобок Пуассона координатных функций $f_{\alpha}, 1 \leqslant \alpha \leqslant 2 m$, невырожденна на всюду плотном в $M$ подмножестве $S$, не включающем $M$, то пара $\left(M,\left.\omega\right|_{M}\right)$ не является симплектическим многообразием, поскольку замкнутая 2-форма $\left.\omega\right|_{M}$ вырождается в точках непустого подмножества $\Theta=M \backslash S$. По теореме Э. Картана [1]

$$
\operatorname{dim} \operatorname{Ker}\left(\left.\omega\right|_{M}\right)=2 m-\operatorname{rk}\left(\left\{f_{\alpha}, f_{\beta}\right\}_{1 \leqslant \alpha, \beta \leqslant 2 m}\right)
$$

следовательно, $\Theta$ состоит из всех точек, в которьх $\operatorname{det}\left(\left\{f_{\alpha}, f_{\beta}\right\}\right)=0$. Если на $M$ задана гладкая функция $f$, то в каждой точке $p$ открытого всюду плотного множества $M \backslash \Theta$ определен вектор $I d f_{p}$ (назьваемьй косым градиентом [1]) такой, что $\omega\left(v, I d f_{p}\right)=v(f)$ для каждого вектора $v \in T_{p} M$. Кроме того, для произвольной гладкой функции $g$ на множестве $M \backslash \Theta$ определена скобка Пуассона $\{f, g\}=I d f(g)$, также являющаяся гладкой функцией. Однако в точках подмножества $\Theta$, называемых особьми, скобки Пуассона и гамильтоновы векторные поля, вообще говоря, не определены. Таким образом, симплектическая геометрия $M$ имеет особенности, которые интересно изучить, поскольку в физических задачах встречаются динамические системы на многообразиях с замкнутой 2-формой $\omega$, являющиеся гамильтоновыми на открытых всюду плотньх подмножествах $\{\operatorname{det}(\omega) \neq 0\}$. Например, они могут возникать при понижении порядка гамильтоновых систем. Таков интегрируемьй случай движения тяжелого магнита в гравитационном и магнитном полях, которьй О.И. Богоявленский нашел при нулевом значении интеграла типа С.В. Ковалевской [2]. Данньй случай был исследован в [3] с точки зрения теории топологической классификации интегрируемьх систем [4]. Его динамика происходит на 4-мерном многообразии с особенностью формы, и особые точки организованы в 3-мерное гладкое подмногообразие. 
В данной работе исследовано предельное поведение гамильтоновьх векторных полей (теоремы 1,2 ) в особых точках, удовлетворяющих естественному условию правильности. В случае, когда все особые точки правильные, найдено условие гладкого продолжения фазового потока, являюшегося гамильтоновым на множестве неособых точек (теорема 1). На основе этого результата получено обобщение теоремы Дарбу [1], [5] о локальном приведении матрицы формы к каноническому виду (теорема 3 ).

ОПРЕДЕЛЕНИЕ 1. Если на гладком $(2 n)$-мерном многообразии $M$ задана замкнутая 2-форма $\omega$, невырожденная в каждой точке из всюду плотного собственного подмножества, то пара $(M, \omega)$ называется симплектическим многообразием с особенностью формы. Каждая точка $x \in M$, в которой $\operatorname{rk}\left(\omega_{x}\right)<\operatorname{dim}(M)$, назьвается особой.

Линейная оболочка множества векторов $A$ обозначается $\mathscr{L}(A)$. В дальнейшем $M^{2 n}$ или $M$ - симплектическое многообразие с особенностью формы, $\Theta$ - множество особых точек, $Z_{p}$ - ядро формы в точке $p \in \Theta$, т.е. $Z_{p}=\operatorname{Ker}\left(\omega_{p}\right)$. Заметим, что $Z_{p}$ всегда имеет четную размерность. Если $\omega(u, v)=0$, то векторы $u$ и $v$ называются косоортогональными. Если на $M$ задана гладкая функция $f$, то $I d f$ - векторное поле на симплектическом многообразии $M \backslash \Theta$. В особых точках косые градиенты, а также скобки Пуассона, вообще говоря, не определены.

ОПРЕДЕЛЕНИЕ 2 . Пусть $f$-гладкая функция на $M$ и $p$-особая точка.

Если для некоторой последовательности точек $p_{n} \notin \Theta$, сходящейся к $p$,

$$
\lim _{n \rightarrow \infty} I d f_{p_{n}}=w \in T_{p} M
$$

то вектор $w$ назьвается предельным положением I $d f$ в точке $p$.

Если для некоторой последовательности точек $p_{n} \notin \Theta$, сходящейся к $p$,

$$
\lim _{n \rightarrow \infty} \mathscr{L}\left(I d f_{p_{n}}\right)=l \neq 0, \quad l \subset T_{p} M
$$

то прямая $l$ называется предельным направлением I $d f$ в точке $p$.

Если при этом $\left|I d f_{p_{n}}\right| \rightarrow+\infty$, то прямая $l$ называется несобственным предельным направлением I df в точке $p$.

Лемма 1. Пусть $p \in M^{2 n}-$ точка на гиперповерхности $P^{2 n-1} \subset \Theta$. Тогда если $Z_{p}$ трансверсально $T_{p} P^{2 n-1}$, а также $\operatorname{dim} Z_{q}=$ const в точках $q \in P^{2 n-1}$, достаточно близких $\kappa p$, то в малой окрестности $U(p)$ существует гладкое интегрируемое распределение $\mathscr{Z}$ такое, что $\mathscr{Z}(q)=Z_{q}$ для всех $q \in P^{2 n-1} \cap U(p)$.

ДокАЗАтЕльство. Пусть $P=P^{2 n-1}$. Сначала докажем, что интегрируемым является гладкое распределение $\zeta: q \mapsto Z_{q} \cap T_{q} P$, определенное на подмногообразии $P \cap O(p)$, где $O(p)$ - достаточно малая окрестность $p$. Предположим, что векторные поля $u$ и $v$ на $P \cap O(p)$ таковы, что $u_{q} \in Z_{q}$ и $v_{q} \in Z_{q}$ для всех $q$. Достаточно доказать, что $[u, v]_{q} \in Z_{q}$ для всех $q$. Так как

$$
L_{u}\left(\left.\omega\right|_{P}\right)=i_{u}\left(\left.d \omega\right|_{P}\right)+d\left(i_{u}\left(\left.\omega\right|_{P}\right)\right)=0,
$$

TO

$$
i_{[u, v]}\left(\left.\omega\right|_{P}\right)=L_{u}\left(i_{v}\left(\left.\omega\right|_{P}\right)\right)-i_{v}\left(L_{u}\left(\left.\omega\right|_{P}\right)\right)=0
$$


где $L$ - производная Ли. Итак, вектор $[u, v]_{q}$ косоортогонален $T_{q} P$, а так как по условию $Z_{q}+T_{q} P=T_{q} M$, то этот вектор косоортогонален $T_{q} M$; следовательно, $[u, v]_{q} \in$ $Z_{q} \cap T_{q} P$.

Фиксируем на гиперповерхности $P$ гладкое трансверсальное поле $z$, так что $z_{q} \in Z_{q}$ при каждом $q$. Тогда интегральные подмногообразия $\zeta$ в фазовом потоке $z$ заметают интегральные подмногообразия искомого распределения $\mathscr{Z}$. Лемма доказана.

Следуюшие два примера показывают, что условие трансверсальности ядра $Z_{p}$ с поверхностью $\Theta$ является сушественным для интегрируемости распределения $\zeta$. В случае, если все $Z_{p}$ касаются $\Theta$, ничего определенного об интегрируемости распределения $\zeta: \Theta \ni q \mapsto Z_{q}$ сказать нельзя.

ПРИмеР 1. Рассмотрим функции $f_{1}(u, v)=u_{1} v_{1}+u_{3}$ и $f_{2}(u, v)=u_{2}+v_{1}^{2}$, где $(u, v) \in \mathbb{R}^{3} \times \mathbb{R}^{3}=\mathbb{R}^{6}$. Уравнение $f_{1}(u, v)=f_{2}(u, v)=0$ определяет 4-мерноеподмногообразие $M \subset \mathbb{R}^{6}$. Пусть $\omega=\left.(d u \wedge d v)\right|_{M}$; тогда $(M, \omega)$ - симплектическое многообразие с особенностью формы, при этом

$$
\Theta=\left\{(u, v) \in M: v_{1}=0\right\}, \quad \operatorname{Ker}\left(\omega_{(u, v)}\right)=\mathscr{L}\left(\frac{\partial}{\partial v_{2}}, \frac{\partial}{\partial v_{3}}-u_{1} \frac{\partial}{\partial u_{1}}\right) .
$$

Ядро формы (размерности 2) касается гиперповерхности $\Theta$ в каждой точке, и распределение $\zeta$ интегрируемо.

ПримеР 2. Пусть $M=\mathbb{R}^{4}$ и $\omega=x_{3} d x_{1} \wedge d x_{2}+x_{2} d x_{1} \wedge d x_{3}-d x_{2} \wedge d x_{4}$; тогда

$$
\Theta=\left\{x \in \mathbb{R}^{4}: x_{2}=0\right\}, \quad \operatorname{Ker}\left(\omega_{x}\right)=\mathscr{L}\left(\frac{\partial}{\partial x_{3}}, \frac{\partial}{\partial x_{1}}-x_{3} \frac{\partial}{\partial x_{4}}\right) .
$$

Ядро формы (размерности 2) касается гиперповерхности $\Theta$ в каждой точке, и распределение $\zeta$ неинтегрируемо.

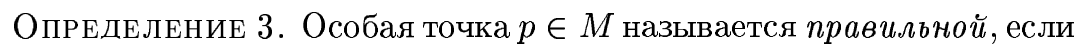

1) существует окрестность $U(p)$ такая, что $\Theta \cap U(p)$ - гладкая гиперповерхность;

2) для всех $q \in \Theta$, достаточно близких к $p$, имеет место $\operatorname{dim} Z_{q}=\operatorname{dim} Z_{p}=2 k$;

3) ядро $Z_{p}$ трансверсально $T_{p} \Theta$;

4 ) в некоторых (и тогда в любых) локальных координатах $x$ найдется частная производная пфаффиана $\mathrm{pf}\left(\omega_{x}\right)$ порядка $2 k-1$, отличная от нуля в точке $x(p)$.

Напомним, что $\mathrm{pf}(\omega)$ - это многочлен с цельми коэффициентами от элементов кососимметрической матрицы $\omega$ такой, что $\operatorname{det}(\omega)=\operatorname{pf}(\omega)^{2}$. Очевидно, что множество правильных особых точек открыто в $\Theta$. В примере $1 \operatorname{pf}\left(\omega_{x}\right)=x_{2}$ и не вьполняется условие 3$)$, а в примере $2 \operatorname{pf}\left(\omega_{(u, v)}\right)=2 v_{1}^{2}$ и не выполняются условия 3$\left.), 4\right)$.

Если $\operatorname{dim} Z_{p}=2$, то условие правильности точки $p$ выглядит так: $Z_{p} \not \subset T_{p} \Theta$ и в некоторых (и тогда в любых) локальных координатах $x$ точка $x(p)$ не является критической для функции $\mathrm{pf}\left(\omega_{x}\right)$. В случае, когда $M$ является подмногообразием совместного регулярного уровня гладких функций $f_{1}$ и $f_{2}$, заданных на симплектическом многообразии $N$, имеем $\Theta=f^{-1}(0)$, где $f=\left.\left\{f_{1}, f_{2}\right\}\right|_{M}$. В этом случае точка $p \in \Theta$ является правильной тогда и только тогда, когда $d\left\{f_{1}, f_{2}\right\}\left(T_{p} M\right) \neq 0$, и хотя бы одно из чисел $\left\{f_{1},\left\{f_{1}, f_{2}\right\}\right\}_{p}$ и $\left\{f_{2},\left\{f_{1}, f_{2}\right\}\right\}_{p}$ отлично от нуля (скобка Пуассона выгисляется в $N$ ).

ПРимеР 3. Применяя последний критерий к случаю Богоявленского [2], [3] получим, что все его особые точки являются правильными. 
Лемма 2. Пусть $p \in M^{2 n}$ - особая точка $u \operatorname{dim} Z_{p}=2 k$. Если в точке $р$ выполнены условия 1)-3) определения 3 , то в некоторой окрестности $U(p)$ существуют локальные координаты $x=\left(x_{1}, \ldots, x_{2 n}\right)$ такие, что

а) зиперповерхность $\Theta \cap U(p)$ задается уравнением $x_{1}=0$;

б) если $2 \leqslant r, s \leqslant 2 n, 1 \leqslant \alpha \leqslant 2 k, 1 \leqslant i \leqslant 2 n$, mo

$$
\omega_{\alpha, i}(x)=\frac{\partial \omega_{r s}}{\partial x_{\alpha}}(x)=0 \quad \text { в каждой точке } \quad x=\left(0, x_{2}, \ldots, x_{2 n}\right) .
$$

ДокАЗАТЕЛЬСТво. Из леммы 1 следует, что в окрестности $p$ существуют локальные координаты $x_{2}, \ldots, x_{2 n}$ многообразия $\Theta$, в которых $(2 k-1)$-мерные координатные поверхности $x_{2}, \ldots, x_{2 k}$ есть интегральные подмногообразия распределения $\zeta$. Очевидно, найдется векторное поле $v$, трансверсальное $\Theta$ такое, что $v_{q} \in Z_{q}$ для всех $q \in \Theta \cap U(p)$. Пусть $\gamma(t)$ - интегральная траектория $v$. Если $\gamma(0)=\left(x_{2}, \ldots, x_{2 n}\right)$ и $\gamma\left(x_{1}\right)=q$, то набор чисел $x_{1}, x_{2}, \ldots, x_{2 n}$ задает искомые координаты точки $q$. При $x_{1}=0$ равенства $\partial \omega_{r s} / \partial x_{\alpha}=0$ следуют из замкнутости формы и равенств $\omega_{\alpha, i}(x)=0$.

ЛЕмма 3. Пусть $p \in M-$ особая точка $u \operatorname{dim} Z_{p}=2 k$. Предположим, что в точке р выполнены условия 1)-3) определения 3. Тогда в произвольных локальных координатах $x$ все частные производные пфаффиана $\operatorname{pf}\left(\omega_{x}\right)$ порядка от 0 до $2 k-2$ включительно равны нулю в точке $x(p)$.

ДокАЗАТЕЛЬСТво. Для произвольной кососимметрической матрищы $\omega_{i j}, 1 \leqslant i$, $j \leqslant 2 n$ :

$$
\operatorname{pf}(\omega)=\sum_{\substack{\sigma=\left(i_{1}, j_{1}, \ldots, i_{n}, j_{n}\right) \in S_{2 n} \\ i_{s}<j_{s}, i_{s}<i_{s+1}}} \operatorname{sgn}(\sigma) \omega_{i_{1} j_{1}} \cdots \omega_{i_{n} j_{n}},
$$

где $S_{2 n}$ - множество перестановок набора $\{1, \ldots, 2 n\}$. Координаты $x_{1}, \ldots, x_{2 n}$ выберем согласно лемме 2. Функция $\mathrm{pf}\left(\omega_{x}\right)$ состоит из слагаемых вида

$$
\omega_{1, j_{1}} \omega_{i_{2}, j_{2}} \cdots \omega_{i_{t}, j_{t}} \omega_{i_{t+1}, j_{t+1}} \cdots \omega_{i_{n}, j_{n}}, \quad \text { где } i_{t} \leqslant 2 k, \quad i_{t+1}>2 k .
$$

Из леммы 2 следует, что при $x_{1}=0$ все элементы $\omega_{i_{2}, j_{2}}, \ldots, \omega_{i_{t}, j_{t}}$ вместе с частными производньми 1-го порядка, а также элемент $\omega_{1, j_{1}}$ равны нулю. Если при этом не равно нулю некоторое слагаемое частной производной порядка $s$ от выражения (1), то это слагаемое с необходимостью содержит множитель

$$
\frac{\partial \omega_{1, j_{1}}}{\partial x_{1}} \frac{\partial^{2} \omega_{i_{2}, j_{2}}}{\partial x_{1}^{2}} \frac{\partial^{2} \omega_{i_{3}, j_{3}}}{\partial x_{1}^{2}} \cdots \frac{\partial^{2} \omega_{i_{t}, j_{t}}}{\partial x_{1}^{2}}
$$

Если $s \leqslant 2 k-2$, то $2 t-1 \leqslant 2 k-2$ и $t<k$. Тогда в выражении (1) найдется не меньше, чем $n-k+1$ сомножителей, у которых $i_{s}, j_{s}>2 k$. Следовательно, найдется не меньше, чем $2(n-k+1)$ различных чисел в промежутке от $2 k+1$ до $2 n$, что невозможно. Итак, $s>2 k-2$. Следовательно, каждая производная от $\operatorname{pf}\left(\omega_{x}\right)$ порядка $s \leqslant 2 k-2$ равна нулю при $x_{1}=0$. Однако производная порядка $2 k-1$ может быть отличной от нуля, и тогда это $\partial^{2 k-1} \operatorname{pf}\left(\omega_{x}\right) / \partial x_{1}^{2 k-1}$, которая равна

$$
\sum_{\substack{\sigma=\left(1, j_{1}, \ldots, i_{n}, j_{n}\right) \in S_{2 n} \\ i_{s}<j_{s}, i_{s}<i_{s+1} \\ i_{k} \leqslant 2 k, i_{k+1}>2 k}} \operatorname{sgn}(\sigma) \frac{\partial \omega_{1, j_{1}}}{\partial x_{1}} \frac{\partial^{2} \omega_{i_{2}, j_{2}}}{\partial x_{1}^{2}} \cdots \frac{\partial^{2} \omega_{i_{k}, j_{k}}}{\partial x_{1}^{2}} \omega_{i_{k+1}, j_{k+1}} \cdots \omega_{i_{n}, j_{n}} .
$$


Если $J(y)$ - определитель матрицы Якоби замены координат $x$ на $y$, то $\operatorname{pf}\left(\omega_{x}\right)=$ $\pm \operatorname{pf}\left(\omega_{y}\right) J(y)$. Следовательно, все частные производные $\operatorname{pf}\left(\omega_{y}\right)$ порядка от 0 до $2 k-2$ равны нулю в точке $y(p)$. Лемма доказана.

Из леммы 3 следует, что если $p$ - правильная особая точка, в которой $\operatorname{dim} Z_{p}=2 k$, и координаты $x$ выбраны согласно лемме 2 , то все частные производные пфаффиана $\operatorname{pf}\left(\omega_{x}\right)$ порядка от 0 до $2 k-1$ включительно равны нулю в точке $x(p)$, кроме, возможно, $\partial^{2 k-1} \mathrm{pf}\left(\omega_{x}\right) / \partial x_{1}^{2 k-1}$. Из доказательства леммы 3 следует пример, проясняюший понятие правильной особой точки.

ПРимеР 4. Пусть в координатах $\left(x_{1}, \ldots, x_{2 k}, p_{1}, \ldots, p_{n-k}, q_{1}, \ldots, q_{n-k}\right)$

$$
\Omega(x, p, q)=\sum_{1 \leqslant i<j \leqslant 2 k} \omega_{i j}\left(x_{1}, \ldots, x_{2 k}\right) d x_{i} \wedge d x_{j}+\sum_{i=1}^{n-k} d p_{i} \wedge d q_{i} .
$$

Предположим, что $\Theta$ - гладкая гиперповерхность, а вырождение формы $\Omega(x, p, q)$ происходит только при обращении в нуль матрицы $\omega(x)$. Ясно, что $\Theta$ представляет собой цилиндр $N^{2 k-1} \times \mathbb{R}^{2 n-2 k}$, где $N^{2 k-1}$ - гладкое подмногообразие в $\mathbb{R}^{2 k}\left(x_{1}, \ldots, x_{2 k}\right)$. Можно считать, что $\Theta$ задается уравнением $x_{1}=0$. При $x_{1}=0$ ядро формы $\Omega$ есть линейная оболочка векторов $\partial / \partial x_{i}, 1 \leqslant i \leqslant 2 k$. Очевидно, что ядро трансверсально цилиндру $\Theta$. Таким образом, особые точки удовлетворяют условиям 1)-3) определения 3. Условие 4) определения 3 эквивалентно невырожденности матрицы

$$
\left(\begin{array}{ccccc}
0 & \frac{\partial \omega_{1,2}}{\partial x_{1}} & \frac{\partial \omega_{1,3}}{\partial x_{1}} & \ldots & \frac{\partial \omega_{1,2 k}}{\partial x_{1}} \\
-\frac{\partial \omega_{1,2}}{\partial x_{1}} & 0 & \frac{\partial^{2} \omega_{2,3}}{\partial x_{1}^{2}} & \ldots & \frac{\partial^{2} \omega_{2,2 k}}{\partial x_{1}^{2}} \\
-\frac{\partial \omega_{1,3}}{\partial x_{1}} & -\frac{\partial^{2} \omega_{2,3}}{\partial x_{1}^{2}} & 0 & \ldots & \frac{\partial^{2} \omega_{3,2 k}}{\partial x_{1}^{2}} \\
\cdots \cdots \cdots \cdots & \ldots \ldots \ldots \ldots & \ldots \\
-\frac{\partial \omega_{1,2 k}}{\partial x_{1}} & -\frac{\partial^{2} \omega_{2,2 k}}{\partial x_{1}^{2}} & -\frac{\partial^{2} \omega_{3,2 k}}{\partial x_{1}^{2}} & \cdots & 0
\end{array}\right)
$$

пфаффиан которой совпадает с $\partial^{2 k-1} \operatorname{pf}\left(\Omega_{(x, p, q)}\right) / \partial x_{1}^{2 k-1}$ при $x_{1}=0$.

Теорема 1. Пусть $f$ - гладкая функиия на симплектическом многообразии $c$ особенностью формы $M^{2 n}$. Для того, чтобы гамильтонова система I df, заданная на $M^{2 n} \backslash \Theta$, имела гладкое продолжение на $M^{2 n}$ необходимо, а если все особие точки правильные, то и достаточно условие:

$$
d f\left(Z_{p}\right)=0 \quad \text { для всех } \quad p \in \Theta .
$$

ДоКАЗАТЕЛЬСТВО. Необходимость: если существует предельное положение $w$ поля $I d f$ в точке $p \in \Theta$, то $\omega(v, w)=d f(v)$ для всех $v \in T_{p} M$; следовательно, $d f\left(Z_{p}\right)=0$.

Пусть теперь $p$ - правильная особая точка. Выберем координаты $x_{1}, \ldots, x_{2 n}$ из леммы 2 так, что $x(p)=0$. Тогда условие $d f\left(Z_{p}\right)=0$ означает, что $\partial f / \partial x_{\alpha}=0$ при $x_{1}=0$ и $1 \leqslant \alpha \leqslant 2 k$. Если $\left(\omega^{i j}\right)$ - матрища, обратная матрище формы $\left(\omega_{i j}\right)$, то

$$
\begin{gathered}
\omega^{i j} \operatorname{pf}(\omega)=-\sum_{\substack{\left(i, j, i_{2}, j_{2}, \ldots, i_{n}, j_{n}\right) \in S_{2 n} \\
i_{s}<j_{s}, i_{s}<i_{s+1}}} \operatorname{sgn}(\sigma) \omega_{i_{2} j_{2}} \ldots \omega_{i_{n} j_{n}}, \\
I d f_{x}^{i}=-\frac{\sum_{j} A_{i j} \partial f / \partial x_{j}}{\sum_{j} \omega_{i j} A_{i j}}
\end{gathered}
$$


где

$$
A_{i j}=\sum_{\substack{\sigma=\left(i, j, i_{2}, j_{2}, \ldots, i_{n}, j_{n}\right) \in S_{2 n} \\ i_{s}<j_{s}, i_{s}<i_{s+1}}} \operatorname{sgn}(\sigma) \omega_{i_{2} j_{2}} \cdots \omega_{i_{n} j_{n}}
$$

Рассмотрим вектор-функцию $F(x)=\left(F^{1}(x), \ldots, F^{2 n}(x)\right)=I d f_{x} \operatorname{pf}\left(\omega_{x}\right)$. Тогда

$$
F^{i}(x)=-\sum_{j=1}^{2 n} A_{i j} \frac{\partial f}{\partial x_{j}}, \quad 1 \leqslant i \leqslant 2 n .
$$

Докажем, что все производные $F(x)$ порядка от 0 до $2 k-2$ равны нулю при $x_{1}=0$.

Фиксируем некоторое $A_{i j} \partial f / \partial x_{j}$ из (3). Каждое $A_{i j}$ состоит из слагаемых вида

$$
\omega_{i_{2}, j_{2}} \cdots \omega_{i_{t}, j_{t}} \omega_{i_{t+1}, j_{t+1}} \cdots \omega_{i_{n}, j_{n}}, \quad \text { где } i_{t} \leqslant 2 k, i_{t+1}>2 k, \quad t \geqslant k
$$

Если $i_{2}>1$, то все $\omega_{i_{2}, j_{2}}, \ldots, \omega_{i_{t}, j_{t}}$ вместе с производными 1-го порядка равны нулю (лемма 2). Так как элементов $\omega_{i_{2}, j_{2}}, \ldots, \omega_{i_{t}, j_{t}}$ не меньше, чем $k-1$, то из всех слагаемых, составляющих частную производную выражения (4) порядка от 0 до $2 k-2$, только слагаемое

$$
\frac{\partial^{2} \omega_{i_{2}, j_{2}}}{\partial x_{1}^{2}} \cdots \frac{\partial^{2} \omega_{i_{k}, j_{k}}}{\partial x_{1}^{2}} \omega_{i_{k+1}, j_{k+1}} \cdots \omega_{i_{n}, j_{n}}, \quad \text { где } i_{k} \leqslant 2 k \quad \text { и } \quad i_{k+1}>2 k
$$

входящее в производную порядка $2 k-2$ по $x_{1}$, быть может, отлично от нуля. Если это так, то поскольку

$$
\left(i, j, i_{2}, j_{2}, \ldots, i_{k}, j_{k}, i_{k+1}, j_{k+1}, \ldots, i_{n}, j_{n}\right)
$$

есть перестановка набора $\{1, \ldots, 2 n\}$, в которой $2 n-2 k$ чисел больше $2 k$ и отлично от $j$, то $j \leqslant 2 k$ и $\partial f / \partial x_{j}=0$. Зафиксируем это и рассмотрим случай $i_{2}=1$.

Если $i_{2}=1$, то из всех слагаемых, составляющих производную выражения (4) порядка от 0 до $2 k-3$, только

$$
\frac{\partial \omega_{1, j_{2}}}{\partial x_{1}} \frac{\partial^{2} \omega_{i_{3}, j_{3}}}{\partial x_{1}^{2}} \cdots \frac{\partial^{2} \omega_{i_{k}, j_{k}}}{\partial x_{1}^{2}} \omega_{i_{k+1}, j_{k+1}} \cdots \omega_{i_{n}, j_{n}}, \quad \text { где } \quad i_{k} \leqslant 2 k \quad \text { и } i_{k+1}>2 k
$$

входящее в производную порядка $2 k-3$ по $x_{1}$, быть может, отлично от нуля. Если это так, то снова $j \leqslant 2 k$ и $\partial f / \partial x_{j}=0$.

Теперь мы можем заключить, что все производные функции $A_{i j} \partial f / \partial x_{j}$ порядка от 0 до $2 k-3$ равны нулю. Следовательно, из производных $A_{i j} \partial f / \partial x_{j}$ порядка $2 k-2$ только производная по $x_{1}$, быть может, отлична от нуля. Тогда все ненулевые слагаемые этой производной являются произведениями на $\partial^{2} f / \partial x_{1} \partial x_{j}$ производных порядка $2 k-3$ по $x_{1}$ от выражений (4) при $i_{2}=1$. Однако поскольку в этом случае $j>1$, из условия теоремы следует $\partial^{2} f / \partial x_{1} \partial x_{j}=0$.

Итак, все производные функции $A_{i j} \partial f / \partial x_{j}$ порядка от 0 до $2 k-2$ равны нулю при $x_{1}=0$, и это же относится к вектор-функции $F(x)$. Следовательно, из всех производных $F(x)$ порядка $2 k-1$ только $\partial^{2 k-1} F / \partial x_{1}^{2 k-1}$ может быть отличной от нуля. 
Теперь разложим $F(x)$ и $\operatorname{pf}\left(\omega_{x}\right)$ в конечные ряды Тейлора и вычислим предел $I d f:$

$$
\begin{aligned}
F(x) & =\frac{\partial^{2 k-1} F(0)}{\partial x_{1}^{2 k-1}} \frac{x_{1}^{2 k-1}}{(2 k-1) !}+\sum_{j=1}^{2 n} \frac{\partial^{2 k} F(\mu x)}{\partial x_{1}^{2 k-1} \partial x_{j}} \frac{x_{1}^{2 k-1} x_{j}}{(2 k) !}, \quad 0<\mu<1, \\
\operatorname{pf}\left(\omega_{x}\right) & =\frac{\partial^{2 k-1} \operatorname{pf}\left(\omega_{0}\right)}{\partial x_{1}^{2 k-1}} \frac{x_{1}^{2 k-1}}{(2 k-1) !}+\sum_{j=1}^{2 n} \frac{\partial^{2 k} \operatorname{pf}\left(\omega_{\eta x}\right)}{\partial x_{1}^{2 k-1} \partial x_{j}} \frac{x_{1}^{2 k-1} x_{j}}{(2 k) !}, \quad 0<\eta<1 .
\end{aligned}
$$

$\lim _{\Theta \not \supset q \rightarrow p} I d f_{q}=\lim _{x \rightarrow 0, x_{1} \neq 0} \frac{F(x)}{\operatorname{pf}\left(\omega_{x}\right)}=\lim _{x \rightarrow 0, x_{1} \neq 0} \frac{F(x) / x_{1}^{2 k-1}}{\operatorname{pf}\left(\omega_{x}\right) / x_{1}^{2 k-1}}=\frac{\partial^{2 k-1} F(0) / \partial x_{1}^{2 k-1}}{\partial^{2 k-1} \operatorname{pf}\left(\omega_{0}\right) / \partial x_{1}^{2 k-1}}$.

Разложение пфаффиана в ряд Тейлора следует из леммы 3 . Итак, предел $I d f$ в точке $p$ существует и гладко зависит от $p$. Теорема доказана.

Для того, чтобы гамильтонова система $I d f$, заданная на множестве $M \backslash \Theta$, имела гладкое (или непрерьвное) продолжение на $M$, вообще говоря, недостаточно условия теоремы 1. Так для функции $f(u, v)=v_{1}$ в примере 1 оно выполнено, однако

$$
\lim _{v_{1} \rightarrow 0}\left|I d f_{(u, v)}\right|=+\infty
$$

Дело в том, что в примере 1 особые точки не являются правильными.

ТЕорема 2. Пусть $f$ - гладкая функиия на симплектическом многообразии $c$ особенностью формы $M^{2 n}$ и $p$ - правильная особая точка. Тогда о предельных полохсениях и предельных направлениях I df в точке $p$ известно следующее.

1. Если $d f_{p} \neq 0$, то существует хотя бы одно предельное направление.

2. Мнохсество несобственных предельных направлений является проективным пространством $\mathbf{P} \mathscr{J}_{p}(f)$, ассочиированным с некоторым подпространством $\mathscr{J}_{p}(f) \subset Z_{p}$.

3. Следующие три утверждения являются әквивалентными:

$$
\mathscr{J}_{p}(f)=0, \quad \mathbf{P} \mathscr{J}_{p}(f)=\varnothing, \quad \exists \lim _{\Theta \not \supset q \rightarrow p} I d f_{q}
$$

4. Если $d f\left(Z_{p}\right) \neq 0$, то предельных положений нет, при этом

$$
\mathbf{P} \mathscr{J}_{p}(f) \neq \varnothing, \quad \mathscr{J}_{p}(f) \subset Z_{p} \cap H_{p}(f), \quad \operatorname{dim} Z_{p} \cap H_{p}(f)=\operatorname{dim} Z_{p}-1,
$$

где $H_{p}(f)$ - гиперплоскость, касательная к подмногообразию $f^{-1}(f(p))$ в точке $p$.

5. Если $d f\left(Z_{p}\right)=0 u d f_{p} \neq 0$, то множество предельных положсений пусто или является плоскостью в $H_{p}(f)$ с направляющим пространством $\mathscr{J}_{p}(f)$, которая не пересекается с $Z_{p} \subset H_{p}(f)$ и косоортогональна $H_{p}(f)$.

6. Если $d f_{p}=0$, то мнохсество предельных положений совпадает $c \mathscr{J}_{p}(f)$ или предельных полохсений нет. 
ДокАЗАТЕльСТво. Если $d f_{p} \neq 0$, то существует хотя бы одно предельное направление, поскольку топологическое пространство

$$
\bigcup_{|q-p| \leqslant \varepsilon} \mathbf{P}\left(T_{q} M^{2 n}\right), \quad \text { где } \varepsilon \rightarrow+0,
$$

является компактом, и $d f_{q} \neq 0$ в достаточно близких к $p$ точках $q \notin \Theta$, в которых соответственно определено направление $I d f_{q}$ (евклидова норма | · вычисляется в произвольных координатах). Обозначим через $\mathscr{J}_{p}(f)$ объединение всех прямых, являющихся несобственными предельными направлениями $I d f$ в точке $p$. Если таковых нет, то положим $\mathscr{J}_{p}(f)=0$. Пусть $q_{n} \notin \Theta$ - последовательность, сходящаяся к $p$. Если $\mathscr{L}(w)-$ несобственноепредельное направлениев точке $p$, то $I d f_{q_{n}}=\lambda_{n} w_{n}$ для некоторойпоследовательности $w_{n} \in T_{q_{n}} M$, сходящейся к $w$. Очевидно, что $\lambda_{n} \rightarrow+\infty$; следовательно, для произвольных $v \in T_{p} M$ и последовательности $v_{n} \in T_{q_{n}} M$, сходящейся к $v$,

$$
\omega(v, w)=\lim _{n \rightarrow \infty} \omega\left(v_{n}, w_{n}\right)=\lim _{n \rightarrow \infty} \frac{1}{\lambda_{n}} d f_{q_{n}}\left(v_{n}\right)=0 \cdot d f_{p}(v)=0 .
$$

Следовательно, $w \in Z_{p}$. Итак, $\mathscr{J}_{p}(f) \subset Z_{p}$.

Пусть $w$ - предельное положение $I d f$ в точке $p$. Ясно, что вектор $w$ косоортогонален $H_{p}(f)$. Так как при $d f\left(Z_{p}\right) \neq 0$ ядро $Z_{p}$ трансверсально $H_{p}(f)$, то $w$ косоортогонален $T_{p} M$; следовательно, $w \in Z_{p}$, что противоречит $d f_{p} \neq 0$. Итак, если $d f\left(Z_{p}\right) \neq 0$, то предельных положений нет, а есть только несобственные предельные направления. Если при этом $\operatorname{dim} Z_{p}=2$, то существует единственное несобственное предельное направление $\mathscr{J}_{p}(f)=Z_{p} \cap H_{p}(f)$, а предельных положений нет. Соотношения между $Z_{p}$ и $H_{p}(f)$, указанные в теореме, элементарны.

По аналогии с теоремой 1 введем координаты $x_{1}, \ldots, x_{2 n}$ из леммы 2 . Пусть $\operatorname{dim} Z_{p}$ $=2 k$ и $I d f_{x}=F(x) / \operatorname{pf}\left(\omega_{x}\right)$. Из доказательства теоремы 1 следует, что если $k>1$, то все частные производные $F(x)$ порядка от 0 до $2 k-4$, а если при этом $d f\left(Z_{p}\right)=0$, то и порядка $2 k-3$, равны нулю при $x_{1}=0$. Если $k \geqslant 1$, то среди производных $F(x)$ порядка $2 k-2$, а если $k>1$ и $d f\left(Z_{p}\right) \neq 0$, то и порядка $2 k-3$, могут быть отличные от нуля в точке $0=x(p)$. Рассмотрим три возможных случая (при $k=1$ возможны только случаи б) и в) ).

a) $\partial^{2 k-3} F(0) / \partial x_{1}^{2 k-3} \neq 0$ :

$$
\begin{gathered}
F(x)=\frac{\partial^{2 k-3} F(0)}{\partial x_{1}^{2 k-3}} \frac{x_{1}^{2 k-3}}{(2 k-3) !}+\sum_{j=1}^{2 n} \frac{\partial^{2 k-2} F(\lambda x)}{\partial x_{1}^{2 k-3} \partial x_{j}} \frac{x_{1}^{2 k-3} x_{j}}{(2 k-2) !}, \quad 0<\lambda<1, \\
\lim _{\Theta \not \supset \rightarrow p} \mathscr{L}\left(I d f_{q}\right)=\lim _{x \rightarrow 0, x_{1} \neq 0} \mathscr{L}\left(\frac{F(x)}{\operatorname{pf}\left(\omega_{x}\right)}\right)=\lim _{x \rightarrow 0, x_{1} \neq 0} \mathscr{L}\left(\frac{F(x) / x_{1}^{2 k-3}}{o\left(x_{1}\right)}\right) .
\end{gathered}
$$

Вектор $\partial^{2 k-3} F(0) / \partial x_{1}^{2 k-3}$ задает единственное предельное направление $I d f$ в точке $p$, которое является несобственным. В данном случае $\operatorname{dim} \mathscr{J}_{p}(f)=1$ и предельных положений нет.

б) $\partial^{2 k-3} F(0) / \partial x_{1}^{2 k-3}=0$, но найдется производная $F(x)$ порядка $2 k-2$, отличная от нуля в точке 0 :

$$
\lim _{\Theta \not \supset q \rightarrow p} \mathscr{L}\left(I d f_{q}\right)=\lim _{x \rightarrow 0, x_{1} \neq 0} \frac{1}{o(1)} \mathscr{L}\left(\frac{\partial^{2 k-2} F(\lambda x)}{\partial x_{1}^{2 k-2}}+\sum_{j=2}^{2 n} \frac{\partial^{2 k-2} F(\lambda x)}{\partial x_{1}^{2 k-3} \partial x_{j}} \frac{x_{j}}{x_{1}}\right) .
$$


Пусть $v_{j}=\partial^{2 k-2} F(0) / \partial x_{1}^{2 k-3} \partial x_{j}$ и $k_{2}, \ldots, k_{2 n}-$ произвольньй набор чисел.

Если $v_{1} \in \mathscr{L}\left(v_{2}, \ldots, v_{2 n}\right)$, то $v_{1}=\sum_{j>1} \alpha_{j} v_{j}$ и вектор $\sum_{j>1} k_{j} v_{j}$ является пределом $I d f_{x}$ при $x_{1} \rightarrow 0$ и $x_{j} \equiv\left(k_{j} \sigma(x)-\alpha_{j}\right) x_{1}$ для всех $j>1$. В частности, при $k_{2}=\cdots=k_{2 n}=0$ получаем 0-вектор; следовательно, $d f_{p}=0$. Полагая $x_{j} \equiv$ $\left(k_{j}-\alpha_{j}\right) x_{1}$ для всех $j>1$, при $x_{1} \rightarrow 0$ получим несобственное предельное направление $\mathscr{L}\left(\sum_{j>1} k_{j} v_{j}\right)$. Итак, в данном случае пространство $\mathscr{J}_{p}(f)=\mathscr{L}\left(v_{2}, \ldots, v_{2 n}\right)$ одновременно является множеством предельных положений и $d f_{p}=0$.

Если $v_{1} \notin \mathscr{L}\left(v_{2}, \ldots, v_{2 n}\right)$, то легко видеть, что все предельные направления несобственные. Полагая $x_{j} \equiv k_{j} x_{1}$ для всех $j>1$, при $x_{1} \rightarrow 0$ в пределе получим предельноенаправление $\mathscr{L}\left(v_{1}+\sum_{j>1} k_{j} v_{j}\right)$, а при $x_{j} \equiv k_{j} \sqrt{x_{1}}$ получим направление $\mathscr{L}\left(\sum_{j>1} k_{j} v_{j}\right)$. В данном случае $\mathscr{J}_{p}(f)=\mathscr{L}\left(v_{1}, \ldots, v_{2 n}\right)$ и предельных положений нет.

в) Все частные производные $F(x)$ порядка от 0 до $2 k-2$ равны нулю в точке $0:$

$$
\lim _{\Theta \not \supset q \rightarrow p} \mathscr{L}\left(I d f_{q}\right)=\lim _{x \rightarrow 0, x_{1} \neq 0} \frac{1}{k+o(1)} \mathscr{L}\left(\frac{\partial^{2 k-1} F(\eta x)}{\partial x_{1}^{2 k-1}}+\sum_{j=2}^{2 n} \frac{\partial^{2 k-1} F(\eta x)}{\partial x_{1}^{2 k-2} \partial x_{j}} \frac{x_{j}}{x_{1}}\right),
$$

где $k=\partial^{2 k-1} \operatorname{pf}\left(\omega_{0}\right) / \partial x_{1}^{2 k-1} \neq 0$. Если $v_{j}=\partial^{2 k-1} F(0) / \partial x_{1}^{2 k-2} \partial x_{j}$, то совокупность направляющих векторов несобственных предельных направлений есть $\mathscr{L}\left(v_{2}, \ldots, v_{2 n}\right)$, а совокупность предельных положений есть плоскость $v_{1}+\mathscr{L}\left(v_{2}, \ldots, v_{2 n}\right)$. Наличие предельных положений $I d f$ в точке $p$ влечет $d f\left(Z_{p}\right)=0$. Итак, в данном случае $\mathscr{J}_{p}(f)=$ $\mathscr{L}\left(v_{2}, \ldots, v_{2 n}\right)$ и плоскость $v_{1}+\mathscr{J}_{p}(f)$ является множеством предельных положений. Ясно, что $v_{1} \in \mathscr{J}_{p}(f)$ эквивалентно $d f_{p}=0$.

Итак, мы рассмотрели все существующие возможности. Из приведенных рассуждений видно, что если $d f\left(Z_{p}\right) \neq 0$, то не имеет места случай в), а если $d f\left(Z_{p}\right)=0$, то не имеет места случай а). Легко построить примеры того, что случай б) совместим с $d f\left(Z_{p}\right) \neq 0$ и с $d f\left(Z_{p}\right)=0$. Сопоставляя все вьшесказанное, получаем утверждения теоремы.

Заметим, что каждая из возможностей, предусмотренных теоремой 2 , реализуется в соответствуюших примерах. При этом размерность пространства $\mathscr{J} p(f)$ может быть различной - от 0 до $\operatorname{dim} Z_{p}$. Следуюший пример показьвает, что многообразия предельных положений и предельных направлений косых градиентов могут быть нелинейными.

ПримеР 5. Пусть $M=\mathbb{R}^{4}$ и $\omega_{x}=x_{1} d x_{1} \wedge d x_{2}+x_{3} d x_{3} \wedge d x_{4}$. В данном случае $\Theta=\left\{x \in \mathbb{R}^{4}: x_{1}=x_{3}=0\right\}$ и особые точки не являются правильными, так как $\operatorname{codim} Z_{x}=2$. Если $f(x)=x_{1} x_{3}$, то конщы векторов, являющихся предельными положениями $I d f$ в точке $x \in \Theta$, описывают в $\mathbb{R}^{2}\left(x_{2}, x_{4}\right)$ гиперболу $x_{2} x_{4}=1$, при этом имеется только два несобственных предельных направления, определяемых осями $x_{2}$ и $x_{4}$.

ТЕоремА 3. Пусть $\left(M^{2 n}, \omega\right)$ - симплектическое многообразие с особенностью формы, $p \in M^{2 n}-$ правильная особая точка $u \operatorname{dim} Z_{p}=2 k$. Тогда в некоторой окрестности р существуют координаты

$$
\left(x_{1}, \ldots, x_{2 k}, p_{1}, \ldots, p_{n-k}, q_{1}, \ldots, q_{n-k}\right),
$$

в которыx

$$
\omega(x, p, q)=\sum_{1 \leqslant \alpha<\beta \leqslant 2 k} \omega_{\alpha \beta}\left(x_{1}, \ldots, x_{2 k}\right) d x_{\alpha} \wedge d x_{\beta}+\sum_{i=1}^{n-k} d p_{i} \wedge d q_{i} .
$$


Матрица $\left(\omega_{\alpha \beta}\right)_{1 \leqslant \alpha, \beta \leqslant 2 k}$ невырожденна при $x_{1} \neq 0$ и обращается в нуль при $x_{1}=0$.

ДокАЗАтЕльство. Следуем идее доказательства теоремы Дарбу из [5]. Всюду $\varepsilon$ обозначает положительное, как угодно малое число. Рассуждаем по индукции. При $n=1$ или $2 k=2 n$ утверждение теоремы очевидно. Предположим, что $n>1$ и $2 k<2 n$. Пусть $O$ - достаточно малая окрестность точки $p$. В окрестности $O$ определено гладкое интегрируемое $2 k$-мерное распределение $\mathscr{Z}$, которое переводит каждое $q \in \Theta \cap O$ в $Z_{q}$ (лемма 1$)$. Вложим в $O$ диск $D^{2 n-2 k}$, так что $p$ - центр диска и $Z_{p}$ трансверсально $D^{2 n-2 k}$. Пусть $f$-гладкая функция на $D^{2 n-2 k}$ такая, что $d f_{p} \neq 0$ и $f(p)=0$. Можно считать, что $D^{2 n-2 k}$ тривиально расслаивается на $(2 n-2 k-1)$-мерные диски $f^{-1}(t)$, где $|t| \leqslant \varepsilon$. Через каждую точку диска $f^{-1}(t)$ проведем интегральное подмногообразие распределения $\mathscr{Z}$ - получится гладкая гиперповерхность $W_{t}$, которая соприкасается с $Z_{q}$ в каждой точке $q \in W_{t} \cap \Theta$. Гладко продолжим функцию $f$ на $O$ так, что $f^{-1}(t)=W_{t}$ при $|t| \leqslant \varepsilon$. Мы получили функцию $f$ на $O$, для которой $d f\left(Z_{q}\right)=0$ для всех $q \in O \cap \Theta$. Из теоремы 1 следует, что в окрестности $O$ определено гладкое векторное поле $I d f$. Ясно, что фазовый поток $I d f$ сохраняет $\omega$ и что $\omega(v, I d f)=v(f)$ для произвольного поля $v$. Пусть $D^{2 n-2 k-1}$ - вложенньй в $D^{2 n-2 k}$ диск с центром $p$, трансверсальньй $(2 n-2 k-1)$-мерному диску $W_{0} \cap D^{2 n-2 k}$. Через каждую точку $D^{2 n-2 k-1}$ проведем интегральное подмногообразие $\mathscr{Z}$ - получится гладкая гиперповерхность $V$, которая соприкасается с $Z_{q}$ в каждой точке $q \in V \cap \Theta$. Выберем диск $D^{2 n-2 k-1}$ так, чтобы он был трансверсален в $D^{2 n-2 k}$ прямой

$$
\left(\mathscr{L}\left(I d f_{p}\right) \oplus Z_{p}\right) \cap T_{p} D^{2 n-2 k}
$$

тогда вектор $I d f_{p}$ трансверсален $V$. Если окрестность $O$ достаточно мала, то каждая точка $q \in O$ может быть получена из некоторой точки $q^{\prime} \in V$ сдвигом на $t$ вдоль интегральной траектории $I d f$, проходящей через $q^{\prime}$. Положим $g(q)=t$. Получаем гладкую функцию $g$ такую, что $g^{-1}(0)=V$. Так как $\{f, g\}(p)=1$, то $d g_{p} \neq 0$. Так как фазовый поток $I d f$ сохраняет $\omega$ и "разносит" $V$ по семейству гладких гиперповерхностей $g^{-1}(t)$, где $|t| \leqslant \varepsilon$, то $d g\left(Z_{q}\right)=0$ для всех $q \in O \cap \Theta$. Следовательно, в окрестности $O$ определено гладкое векторное поле $I d g$. Так как $\{f, g\} \equiv 1$, ковекторы $d g_{p}$ и $d f_{p}$ линейно независимы.

Пусть $M^{\prime}=\{q \in O: f(q)=g(q)=0\}$ и $\omega^{\prime}$ - ограничение формы $\omega$ на $(2 n-2)$-мерное подмногообразие $M^{\prime}$. Так как $M^{\prime}=W_{0} \cap V$, то $Z_{q} \subset T_{q} M^{\prime}$ для всех $q \in M^{\prime}$. Линейная оболочка векторов $I d f_{p}$ и $I d g_{p}$ трансверсальна $T_{p} M^{\prime}$, так как если $v=\lambda I d f_{p}+\mu I d g_{p} \in$ $T_{p} M^{\prime}$, то $d f(v)=-\mu=0$ и $d g(v)=\lambda=0$. Так как векторы $I d f_{q}$ и $I d g_{q}$ косоортогональны $T_{q} M^{\prime}$ и касаются $\Theta$ при $q \in \Theta$, то $M^{\prime}$ трансверсально $\Theta$ (в противном случае $(2 n-1)$-мерное касательноепространство к $\Theta$ инцидентно $(2 n-2)$-мерному касательному пространству к $M^{\prime}$ и, одновременно, трансверсально последней 2-мерной линейной оболочке векторов $I d f$ и $I d g$, что абсурдно). Следовательно, $\left(M^{\prime}, \omega^{\prime}\right)$ - почти симплектическое многообразие, причем $\Theta_{M^{\prime}}=\Theta \cap M^{\prime}$, и для всех $q \in \Theta_{M^{\prime}}$ ядро $\omega_{q}^{\prime}$ совпадает c $Z_{q}$.

Проверим, что точка $p$ правильная в $M^{\prime}$. Ясно, что $\Theta_{M^{\prime}}$ - гладкая гиперповерхность в $M^{\prime}$ и $\operatorname{dim} Z_{q}=2 k$ для всех $q \in \Theta_{M^{\prime}}$. Так как $Z_{p} \not \subset T_{p} \Theta$, то тем более $Z_{p} \not \subset T_{p} \Theta_{M^{\prime}}$. Осталось проверить условие 4) определения 2 . Подмногообразие $M^{\prime}$, “дрейфуя” в потоке $I d g$, заметает некоторую гиперповерхность $S$. В свою очередь $S$ под действием потока $I d f$ заметает окрестность точки $p$. Обозначим $I d f$ через $v_{2 n-1}$ и $I d g$ через $v_{2 n}$. 
Тождество $\{f, g\} \equiv 1$ влечет

$$
\left[v_{2 n-1}, v_{2 n}\right]=[I d f, I d g]=\operatorname{sgrad}\{f, g\}=\operatorname{sgrad}(1)=0
$$

в каждой точке $O \backslash \Theta$; следовательно, $\left[v_{2 n-1}, v_{2 n}\right]=0$ на $O$. Поскольку $\left[v_{2 n-1}, v_{2 n}\right] \equiv 0$, линейная оболочка $\mathscr{L}\left(I d f_{p}, I d g_{p}\right)$ трансверсальна $M^{\prime}$ и множество $\Theta$ инвариантно относительно $v_{2 n-1}$ и $v_{2 n}$, то для любых координат $\left(y_{1}, \ldots, y_{2 n-2}\right)$ на $M^{\prime}$ существуют координаты $\left(y_{1}, \ldots, y_{2 n-2}, y_{2 n-1}, y_{2 n}\right)$ на $O$ такие, что

а) векторы $v_{2 n-1}$ и $v_{2 n}$ являются касательными к координатным линиям $y_{2 n-1}$ и $y_{2 n}$;

б) подмногообразие $M^{\prime}$ задается уравнением $y_{2 n-1}=y_{2 n}=0$;

в) гиперповерхность $O \cap \Theta$ задается уравнением $y_{1}=0$.

Если $y^{\prime}=\left(y_{1}, \ldots, y_{2 n-2}\right)$ - вектор координат $M^{\prime}$ и $\left(y^{\prime}, 0\right)=\left(y_{1}, \ldots, y_{2 n-2}, 0,0\right)$, то

$$
\operatorname{det}\left(\omega_{\left(y^{\prime}, 0\right)}\right)=\operatorname{det}\left(\omega_{y^{\prime}}^{\prime}\right) \operatorname{det}\left(\begin{array}{cc}
0 & 1 \\
-1 & 0
\end{array}\right)
$$

поскольку $\omega\left(v_{2 n-1}, v_{2 n}\right)=1$. Следовательно, $\operatorname{pf}(\omega)_{\left(y^{\prime}, 0\right)}= \pm \operatorname{pf}\left(\omega^{\prime}\right)_{y^{\prime}}$. Очевидно, что координатная линия $y_{1}=t$ в $M^{\prime}$, проходящая через точку $p$, является таковой и в $M$. Согласно лемме 3 условие 4) определения 2 сводится к тому, что ограничение функции $\operatorname{pf}(\omega)_{y}$ на координатную линию $y_{1}=t$ имеет отличную от нуля производную порядка $2 k-1$ в точке $y_{1}(p)$. Следовательно, данное условие вьполняется (или не вьполняется) в $M$ и $M^{\prime}$ одновременно. Итак, особая точка $p$ многообразия $M^{\prime}$ является правильной.

Согласно индуктивному предположению на многоообразии $M^{\prime}$ существуют координаты $\left(x^{\prime}, p^{\prime}, q^{\prime}\right)=\left(x_{1}, \ldots, x_{2 k}, p_{1}, \ldots, p_{n-k-1}, q_{1}, \ldots, q_{n-k-1}\right)$, в которых

$$
\omega^{\prime}\left(x^{\prime}, p^{\prime}, q^{\prime}\right)=\sum_{1 \leqslant \alpha<\beta \leqslant 2 k} \omega_{\alpha \beta}\left(x_{1}, \ldots, x_{2 k}\right) d x_{\alpha} \wedge d x_{\beta}+\sum_{i=1}^{n-k-1} d p_{i} \wedge d q_{i} .
$$

При этом $\omega_{\alpha \beta}(x)=0$ при $x_{1}=0$ и $\operatorname{det}\left(\omega_{\alpha \beta}(x)\right) \neq 0$ при $x_{1} \neq 0$. Введем в $O$ координаты $\left(x_{1}, \ldots, x_{2 k}, p_{1}, \ldots, p_{n-k-1}, y_{2 n-1}, q_{1}, \ldots, q_{n-k-1}, y_{2 n}\right)$. Обозначим координаты $y_{2 n-1}$ и $y_{2 n}$ через $p_{n-k}$ и $q_{n-k}$. Все координатные поверхности вида $y_{2 n-1}=$ const $_{1}$, $y_{2 n}=$ const $_{2}$ получаются из $M^{\prime}$ последовательными сдвигами в фазовых потоках $I d g$ и $I d f$, при которых каждая $i$-я координатная линия $M^{\prime}$ сдвигается в $i$-ю координатную линию $O$. При этом $\Theta_{M^{\prime}}$ заметает $\Theta$. Поскольку данные потоки сохраняют форму $\omega$ и поскольку $\omega\left(\partial / \partial p_{n-k}, \partial / \partial q_{n-k}\right)=1$, то $\omega$ имеет вид (4). Теорема доказана.

СледСТВИЕ 1. Пусть $p \in M^{2 n}-$ правильная особая точка $u \operatorname{dim} Z_{p}=2 k$. Тогда в некоторой окрестности $p$ существуют координаты $\left(x_{1}, \ldots, x_{2 n}\right)$, в которых матрича скобок Пуассона координатных функиий имеет вид

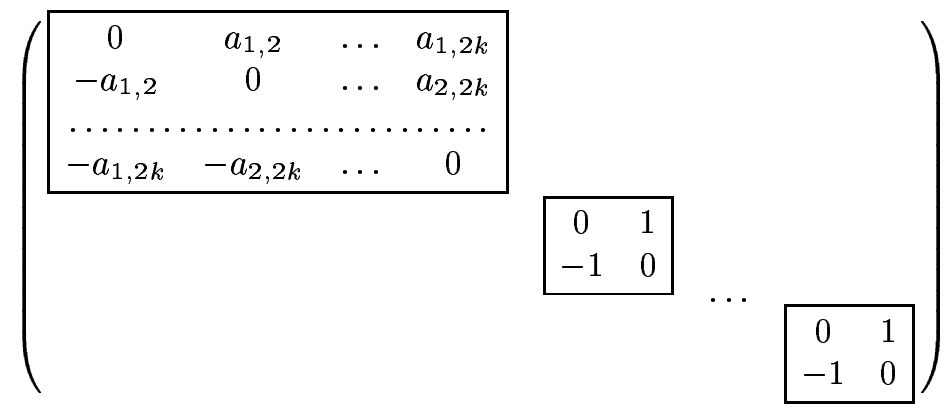


для некоторой матрицы $a(x)=\left(a_{i, j}\right)_{1 \leqslant i, j \leqslant 2 k}$, невырожденной при $x_{1} \neq 0$. Кажсдая из функиий $a_{i, j}(x)$ является гладкой при $x_{1} \neq 0$ и не зависит от координат $x_{2 k+1}, \ldots, x_{2 n}$. Некоторые из функций $a_{i, j}(x)$ (возможсно все) не определены при $x_{1}=0$. В каждой строке и каждом столбие матрицы а $(x)$ найдется әлемент $a_{i, j}$ такой, что

$$
\limsup _{x \rightarrow x(p), x_{1} \neq 0}\left|a_{i, j}\left(x_{1}, \ldots, x_{2 k}\right)\right|=+\infty .
$$

ДокАЗАТЕЛЬСТво. Блочно-диагональный вид (5) матрица Пуассона имеет в координатах $x$, построенных в теореме 3 , если их соответствующим образом перенумеровать. Пусть $x(p)=0 \in \mathbb{R}^{2 n}$. Элементы $a_{i, j}$ зависят только от $x_{1}, \ldots, x_{2 k}$, поэтому можно считать, что $2 k=2 n$. Пусть $1 \leqslant i, j \leqslant 2 k$. Так как $d x_{i} \neq 0$ для каждой координатной функции $x_{i}$, то ни одно из $I d x_{i}$ не имеет предельных положений в точке $p$, однако имеет несобственные предельные направления, т.е. $\mathscr{J}_{p}\left(x_{i}\right) \neq 0$ (теорема 2 ). Так как $a_{i, j}(x)=d x_{j}\left(I d x_{i}\right)$ при $x_{1} \neq 0$, то

$$
\limsup _{x \rightarrow 0, x_{1} \neq 0}\left|a_{i, j}(x)\right|<+\infty
$$

влечет $\mathscr{J}_{p}\left(x_{i}\right) \subset H_{p}\left(x_{j}\right)=T_{p}\left\{x_{j}=0\right\}$. Следовательно, если в $i$-й строке матрицы $a(0)$ все элементы удовлетворяют условию (6), то

$$
\mathscr{J}_{p}\left(x_{i}\right) \subset \bigcap_{j=1}^{2 k} H_{p}\left(x_{j}\right)=0,
$$

что невозможно, поскольку $\mathscr{J}_{p}\left(x_{i}\right) \neq 0$. Это же относится и к произвольному столбцу, поскольку матрица кососимметрична. Следствие доказано.

Из замкнутости формы $\omega$ следует, что увеличить число блоков на главной диагонали матрицы (5) невозможно. Следствие 1 дает некоторое представление о сингулярном поведении скобки Пуассона в правильной особой точке.

Автор глубоко признателен академику А. Т. Фоменко за внимание к работе и профессору А. В. Болсинову за критические замечания, которые помогли сформулировать ключевое понятие правильной особой точки.

\section{СПИСОК ЦИТИРОВАННОЙ ЛИТЕРАТУРЫ}

[1] Фоменко А.Т. Симплектическая геометрия. Методы и приложения. М.: МГУ, 1988.

[2] Богоявленский О.И.Интегрируемые уравнения Эйлера на алгебрах Ли, возникающие в задачах математической физики // Изв. АН СССР. Сер. матем. 1984. Т. 48. № 5. С. 883-938.

[3] Зотьев Д. Б. Fomenko-Zieschang invariant in the Bogoyavlenskyi integrable case // Regular\&chaotic dynamics. 2000. V. 5. № 4. P. 437-458.

[4] Болсинов А. В., Фоменко А. Т. Интегрируемые гамильтоновы системы. Топология. Геометрия. Классификация. Ижевск: Изд. дом "Удмуртский университет", 1999.

[5] Арнольд В.И. Математические методы классической механики. М.: Наука, 1979.

Волгоградский государственный технический университет

E-mail : zotev@fizmat.vspu.su 